\title{
Abdominal distension and diarrhea as the main symptoms of primary amyloidosis: A case report and literature review
}

\author{
JIAN ZHANG, CHUXIAO SHAO, JINDE ZHU, CHAOYONG TU and XINLIANG LV \\ Department of Hepatopancreatobiliary Surgery, The Fifth Affiliated Hospital of Wenzhou Medical University, \\ Lishui, Zhejiang 323000, P.R. China
}

Received March 24, 2015; Accepted October 29, 2015

DOI: 10.3892/etm.2016.3093

\begin{abstract}
Amyloidosis is a systemic disease caused by the accumulation of extracellular protein into amyloid deposits. Due to the involvement of a variety of organs and tissues, the disease has no specific clinical features and a high rate of misdiagnosis. The present study describes one case of primary amyloidosis, as confirmed by biopsies of rectal and renal tissues using Congo red staining, demonstrating that abdominal distension and diarrhea were the main symptoms of disease. The current case is also discussed within the context of a review of the associated literature.

\section{Introduction}

Amyloidosis was first described in 1854 by the pathologist Rudolph Virchow, and is a systemic disease that results from the tissue deposition of misfolded proteins, causing the progressive failure of organs (1). A total of 6 types of amyloidosis exist; these are primary, secondary, hemodialysis-associated, hereditary, senile and localized (2). The most common of these 6 types is primary amyloidosis, the prevalence of which is $\sim 4$ in 10,000 cases (3). Primary amyloidosis, in which fibrils are made up of a monoclonal immunoglobulin light chain, is the most common and the most severe form of amyloidosis. The present study presents a case of primary amyloidosis with digestive system involvement in the form of abdominal distension and diarrhea.
\end{abstract}

\section{Case report}

A 51-year-old male patient was referred to the Department of Hepatopancreatobiliary Surgery, The Fifth Affiliated Hospital of Wenzhou Medical University (Lishui, Zhejiang, China) on March 5, 2014 with a 5-month history of abdominal distension

Correspondence to: Professor Chuxiao Shao, Department of Hepatopancreatobiliary Surgery, The Fifth Affiliated Hospital of Wenzhou Medical University, 289 Kuocang Road, Liandu, Lishui, Zhejiang 323000, P.R. China

E-mail: scx1818@126.com

Key words: primary amyloidosis, abdominal distension, diarrhea and diarrhea, excreting yellow, loose stool 4-5 times a day that contained no pus, blood or mucus. The patient presented with no abdominal pain, acid reflux, chest tightness, difficulty breathing, chills or fever, and was otherwise healthy, indicating no signs of osteomyelitis, myeloma, rheumatoid arthritis or an associated family history of these conditions.

A physical examination revealed no significant abnormalities; the patient was negative for macroglossia (Fig. 1), which is indicative of gastrointestinal tract involvement, and results for the complete blood count, echocardiogram, erythrocyte sedimentation rate, and basic metabolic and liver function tests were all normal. However, serum and urine protein electrophoresis tests indicated an abnormal $\kappa / \lambda$ ratio of 0.7 (normal range, 1.5-3.0). B-mode ultrasonography demonstrated changes to the kidneys (enhanced enchogenicity and renal parenchyma) and ascites; gastroscopy also demonstrated an ulcerous duodenal bulb (S1 gastric ulcer stage) and superficial gastritis. A needle biopsy and subsequent microscopic evaluation of the kidney revealed Congo red-positive material deposited within the mesangium and renal interstitium (Fig. 2), enabling a histopathological diagnosis of renal amyloidosis. A colonoscopy revealed 'cobblestone' patterns of ulcerations in the colon (Fig. 3), and the interstitium of the mucoderm and submucosa surrounding the blood vessels of the rectal tissues demonstrated positive staining for Congo red (Fig. 4). From the integration of these physical, laboratory and histological examinations, a diagnosis of localized primary amyloidosis was formed.

The patient was initially diagnosed with nephrosis, duodenal bulb ulcers and functional enteritis prior to amyloidosis. Following a diagnosis, the patient was treated with bifidobacteria ( $1 \mathrm{~g}$ tid PO) to modulate the intestinal microflora, $\alpha$-keto acid tablets ( $2.52 \mathrm{~g}$ tid $\mathrm{PO}$ ) to protect the kidneys by reducing the synthesis of urea and the accumulation of toxic uremic products, omeprazole enteric-coated capsules (20 mg qd PO) to protect the gastrointestinal tract from acidic damage, and enteritis syrup (10 ml tid PO) and composite Lactobacillus capsules (0.33 g tid PO) to treat the diarrhea. Following treatment with these drugs, there was no marked improvement of symptoms, and the patient declined the continuation of treatment.

\section{Discussion}

Amyloidosis is a rare disease, and its prognoses and clinical manifestations are broad. Common symptoms of primary 


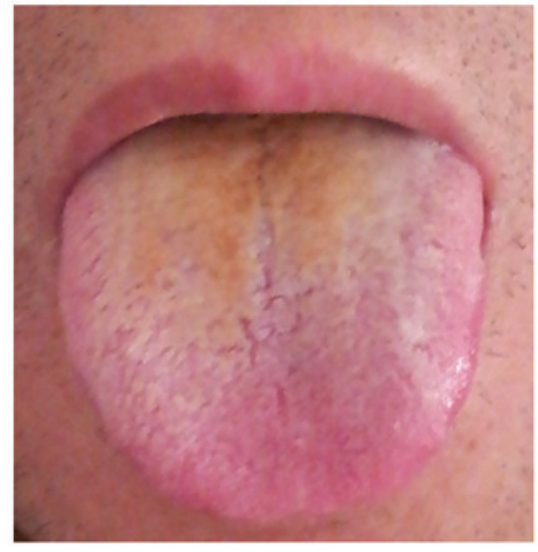

Figure 1. No macroglossia of the tongue was observed.

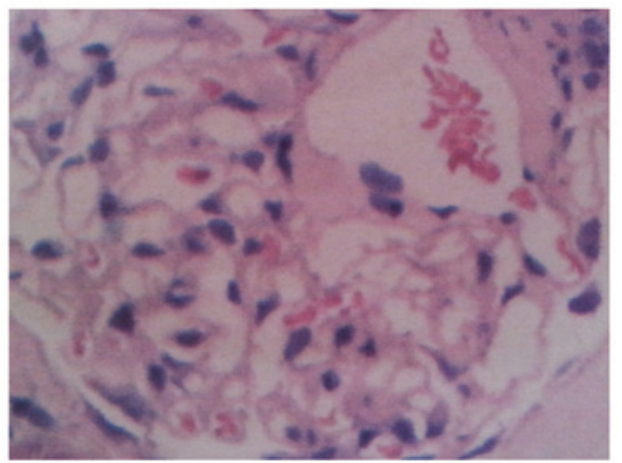

Figure 2. Positive Congo red staining of deposits within the mesangium and interstitium of renal tissue.

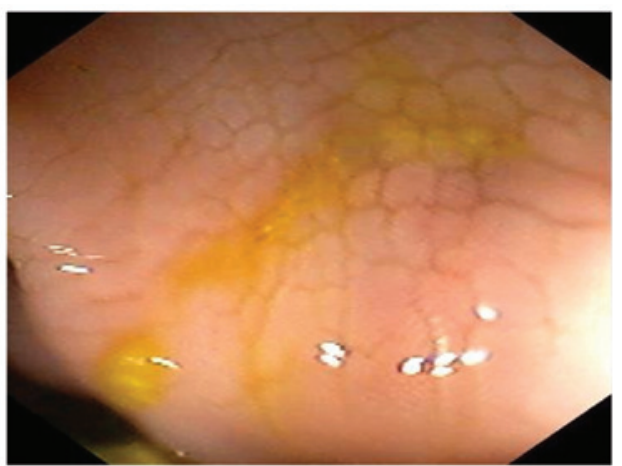

Figure 3. Colonoscopy image demonstrating the 'cobblestone' appearance of the ulcerations.

amyloidosis include fatigue, dyspnoea and frequently, kidney disease; patients often develop proteinuria, which progresses into renal failure (4). Cardiac involvement is also prevalent; heart failure and arrhythmia are the predominant cause of mortality from amyloidosis (5). Involvement of other organs occurs as follows: Hepatic deposition causes liver enlargement and increased alkaline phosphatase levels; depositions on the spleen or lungs leads to impairment of spleen function or respiratory failure, respectively; and cutaneous depositions trigger the formation of bilateral periorbital purpura, papules and plaques (6). Macroglossia typically occurs as a result of oral lesions, and leads to sleep apnea and dysphasia. The
A

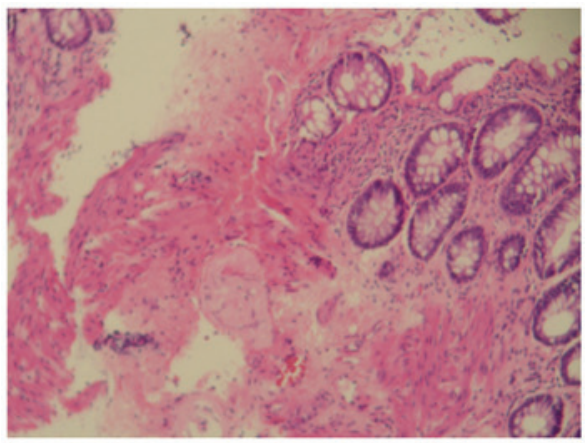

B

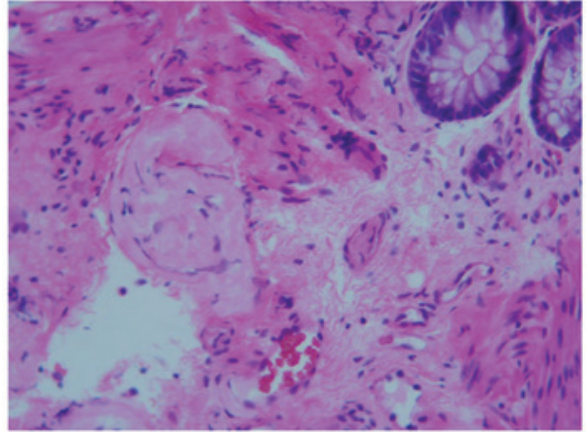

C

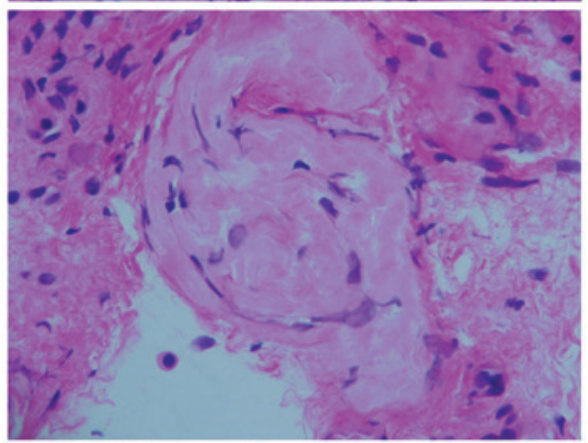

D

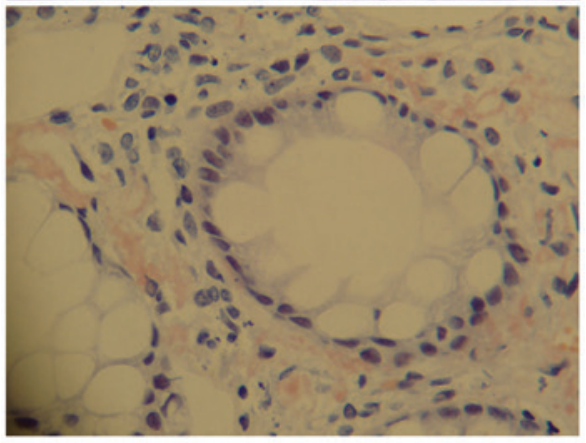

Figure 4. Pathology of rectal mucosal tissue. The interstitium of the mucoderm and submucosa surrounding the blood vessels with hematoxylin and eosin staining at (A) x100, (B) x200 and (C) x400 magnification. (D) Congo red-positive staining, $\mathrm{x} 400$ magnification.

clinical manifestations of gastrointestinal involvement are determined by the location and quantity of protein deposition. Gastric and duodenal involvement can cause symptoms including nausea, abdominal pain and hematemesis, and small intestinal involvement can result in symptoms such as diarrhea, steatorrhea, gastrointestinal bleeding and obstruction.

The diagnosis of amyloidosis requires a combinatorial approach of clinical and imaging examinations and pathological diagnosis. Previous studies have revealed that positron emission tomography/computed tomography (CT) using 
${ }^{18} \mathrm{~F}$-fluorodeoxyglucose $\left({ }^{18} \mathrm{~F}-\mathrm{FDG}\right)$ as an imaging agent can be used to detect the lung nodules of amyloid deposition, as these nodules tend to absorb FDG (7), and increased ${ }^{18}$ F-FDG uptake is also reported in the livers of patients with primary amyloidosis (8). Hypoperfusion of the spleen, demonstrated using an enhanced CT scan, may also enable the diagnosis of systemic amyloidosis (9). All the aforementioned non-invasive techniques aid the diagnosis of amyloidosis, but the pathological examination of organs, such as through use of Congo red staining of the biopsy, remains the most effective way to confirm this diagnosis (10).

There are no specific treatments for amyloidosis at present, the aims of which would be to eliminate the light chain of the misfolding amyloid protein, and to support the function of affected organs. Dexamethasone has replaced prednisone in combination treatments with melphalan due to the greater hematological response to this regimen (11). Intravenous administration of high-dose melphalan combined with autologous hematopoietic stem cell transplantation can also improve the patient survival rate (11). Due to the broad nature of symptoms, individualized treatments of the target organs may also be used; continuous peritoneal dialysis and kidney transplants are useful in renal lesions, for instance (12), and diuretics can be used to treat patients with congestive heart failure (13).

The misdiagnosis rate of amyloidosis is high, due to the fact that the clinical manifestations of primary amyloidosis are complex, varied, and lack specificity, which often delays diagnosis. In this present case, abdominal distension and diarrhea as the primary symptoms, although these are not common symptoms. In clinical settings disease symptoms should be analyzed systematically, and rare diseases should be considered following the exclusion of common diseases to avoid delays in diagnosis.

\section{References}

1. Sipe JD and Cohen AS: Review: History of the amyloid fibril. J Struct Biol 130: 88-98, 2000.

2. Bhat A, Selmi C, Naguwa SM, Cheema GS and Gershwin ME: Currents concepts on the immunopathology of amyloidosis. Clin Rev Allergy Immunol 38: 97-106, 2010.

3. Gertz MA, Lacy MQ and Dispenzieri A: Amyloidosis: Recognition, confirmation, prognosis, and therapy. Mayo Clin Proc 74: 490-494, 1999.

4. Anupama YJ and Vankalakunti M: Rapidly progressive glomerulonephritis in a patient with renal amyloidosis: Case report and review of the literature. Indian J Nephrol 22: 377-380, 2012.

5. Gertz MA, Comenzo R, Falk RH, Fermand JP, Hazenberg BP, Hawkins PN, Merlini G, Moreau P, Ronco P, Sanchorawala V, et al: Definition of organ involvement and treatment response in immunoglobulin light chain amyloidosis (AL): A consensus opinion from the 10th International Symposium on Amyloid and Amyloidosis, Tours, France, 18-22 April 2004. Am J Hematol 79: 319-328, 2005.

6. Desport E, Bridoux F, Sirac C, Delbes S, Bender S, Fernandez B, Quellard N, Lacombe C, Goujon JM, Lavergne D, et al; Centre national de référence pour l'amylose AL et les autres maladies par dépôts d'immunoglobulines monoclonales: Al amyloidosis. Orphanet J Rare Dis 7: 54, 2012.

7. Seo JH, Lee SW, Ahn BC and Lee J: Pulmonary amyloidosis mimicking multiple metastatic lesions on F-18 FDG PET/CT. Lung Cancer 67: 376-379, 2010.

8. Son YM, Choi JY, Bak CH, Cheon M, Kim YE, Lee KH and Kim BT: 18F-FDG PET/CT in primary AL hepatic amyloidosis associated with multiple myeloma. Korean J Radiol 12: 634-637, 2011.

9. Mainenti PP: RE: Imaging features of hepato-splenic amyloidosis at PET/CT. Korean J Radiol 13: 368-369, 2012.

10. Suzuki K: Diagnosis and treatment of multiple myeloma and AL amyloidosis with focus on improvement of renal lesion. Clin Exp Nephrol 16: 659-671, 2012.

11. Merlini G, Seldin DC and Gertz MA: Amyloidosis: Pathogenesis and new therapeutic options. J Clin Oncol 29: 1924-1933, 2011.

12. Gude D, Chennemsetty S, Jha R and Narayan G: Primary amyloidosis treated with continuous ambulatory peritoneal dialysis. Saudi J Kidney Dis Transpl 23: 1285-1287, 2012.

13. Falk RH: Cardiac amyloidosis: A treatable disease, often overlooked. Circulation 124: 1079-1085, 2011. 